\title{
DETERMINAÇÃO DOS COEFICIENTES DE ATIVIDADE NA DILUIÇÃO INFINITA DE COMPOSTOS ORGÂNICOS EM [BMIM] [MESO 4 ] POR HS-SPME/GC-FID NAS TEMPERATURAS DE 298.15, 313.15, 333.15 E 353.15 K
}

\author{
A.M. ELIAS ${ }^{1}$ e G.L.V. COELHO ${ }^{1}$ \\ ${ }^{1}$ Universidade Federal Rural do Rio de Janeiro, Departamento de Engenharia Química \\ E-mail para contato: coelho@ufrrj.br
}

\begin{abstract}
RESUMO - Uma nova metodologia utilizando a microextração por fase sólida através da extração no headspace (SPME-HS) foi utilizada para determinar os coeficientes de atividade na diluição infinita de dois álcoois (etanol e 1-butanol) em liquido iônico. $\mathrm{O}$ principal objetivo do trabalho foi validar a implementação de uma técnica rápida e de baixo custo. Os coeficientes de atividade na diluição infinita tem grande importância na indústria química principalmente em processos de separação de misturas liquidas. Os resultados foram comparados com metodologias já consolidadas na literatura, como o esgotamento com gás inerte apresentando desvios que variaram de 0.8 a $23 \%$. Os líquidos iônicos, como o metilsulfato de 1-butil-3-metilimidazolíneo, vêm despertando um grande interesse em processos de separação devido a excelentes características como baixa pressão de vapor, estabilidade térmica, boa solubilidade e baixa corrosibilidade.
\end{abstract}

\section{INTRODUÇÃO}

A separação de misturas em componentes puros é um dos mais importantes processos da indústria química. A modelagem do processo térmico requer um estudo detalhado das condições de equilíbrio do sistema, afim de se determinar os parâmetros termodinâmicos do sistema, como propriedades de excesso e atividades dos componentes da mistura. O coeficiente de atividade em diluição infinita tem como objetivo definir o correto dimensionamento do processo, visto que em situações de extrema diluição (p. ex., final do processo de destilação), a obtenção de componentes puros se torna mais difícil. Outra função é definir qual o melhor solvente a ser utilizado na remoção de um dado componente de uma solução em maior quantidade que os outros, parâmetro denominado seletividade, definido como a relação entre dois coeficientes de atividade em diluição infinita dos solutos no mesmo solvente em uma mesma temperatura (Furtado e Coelho, 2010; Krummen et al, 2002).

Os solventes seletivos apresentam uma importante função na separação de soluções, pois podem promover uma expressiva separação, em alguns casos, até mesmo realizar a quebra de azeótropos. Líquidos iônicos têm atraído à atenção dos pesquisadores, pois são considerados opções mais ecológicas que solventes orgânicos voláteis convencionais. Normalmente são menos poluentes, possuem pressão de vapor desprezível, podem ser recicláveis, possuem estabilidade térmica, boa solubilidade e baixa corrosibilidade. São descritos na literatura como cátions volumosos, orgânicos e 

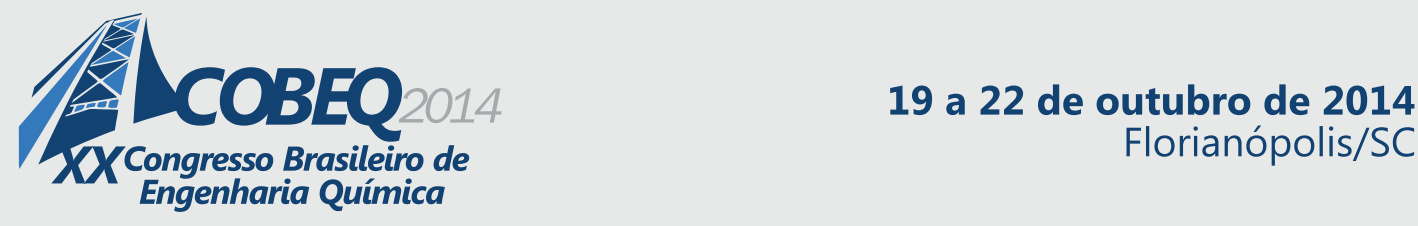

Florianópolis/SC

de baixa simetria. Possuem ponto de fusão abaixo de $100^{\circ} \mathrm{C}$. Os principais cátions são os imidazolíneo e piridíneo. $\mathrm{O}$ ânions são geralmente poliatômicos e inorgânicos, como o $\mathrm{CH}_{3} \mathrm{SO}_{4}^{-}, \mathrm{BF}_{4}^{-}$ (Greaves e Drummond, 2008; Huang et al., 2008).

Os coeficientes de atividade em diluição infinita podem ser determinados por vários métodos como por cromatografia gás-liquida, ebuliometria, esgotamento com gás inerte e SPME. Este último foi empregado com sucesso na determinação de parâmetros termodinâmicos em sistemas orgânicos, com desvios aceitáveis, se apresentando como um processo eficiente e de baixo custo (Fonseca e Coelho, 2007; Furtado e Coelho, 2010; Furtado e Coelho, 2012).

Neste estudo os coeficiente de atividade na diluição infinita de 2 solutos foram determinadas em temperaturas que variaram de $298.15 \mathrm{~K}$ a $353.15 \mathrm{~K}$ em metilsulfato de 1-butil-3-metilimidazolíneo utilizando a metodologia de headspace/SPME. Zhang e Pawliszyn (1996) desenvolveram uma metodologia alternativa, de fácil implementação utilizando a SPME aliada a cromatografia para a determinação do coeficiente de atividade. A partir deste equacionamento foi possível desenvolver uma modelagem termodinâmica que permite o cálculo do coeficiente de atividade em diluição infinita através da análise de headspace (Furtado e Coelho, 2010; Furtado e Coelho, 2012)

\section{PARTE EXPERIMENTAL}

Os solutos(1) utilizados foram obtidos da Vetec Química Fina Ltda sendo: etanol, e 1-butanol com pureza superior a $99.4 \%$, verificado por cromatografia gasosa. O solvente(2) utilizado, o metilsulfato de 1-butil-3-metilimidazolíneo foi obtido da Basf com pureza superior a 95\%. A fibra de PDMS (polidimetilsiloxano) de espessura $100 \mu \mathrm{m}$ foi obtida da Supelco. O cromatógrafo a gás utilizado no experimento foi um GC-2010 Shimadzu equipado com detector de ionização em chama $(\mathrm{FID}=$ Flame Ionization Detector), uma coluna capilar HP-Innowax (polietileno glicol reticulado; crosslinked PEG) de dimensões $60 \mathrm{~m}$ x $0.32 \mathrm{~mm}$ x $0.25 \mu \mathrm{m}$, um liner da SGE Analytical Science Pty Ltd, de diâmetro interno $0.75 \mathrm{~mm}$ (próprio para SPME) e hélio ultrapuro (99.999\%) como gás de arraste. Frascos âmbar de $40 \mathrm{~mL}$ com septo de PTFE/silicone serviram para o confinamento e estudo das misturas líquidas.

O LI (líquido iônico), antes da sua utilização, foi purificado através de evaporação rotativa na pressão de 2 mbar em temperatura constante de $55^{\circ} \mathrm{C}$ por 5 dias. Para confirmar a pureza do LI, foi realizado por SPME uma análise do headspace de $5 \mathrm{~mL}$ do LI em um frasco de $40 \mathrm{~mL}$, sendo confirmado a pureza do composto tratado devido a inexistência de picos cromatográficos.

A curva de calibração para o etanol e para o 1-butanol foi construída injetando-se volumes precisos de $1 \mu \mathrm{L}$ de solução com concentrações variadas dos componentes em solventes (xileno e etanol, respectivamente, ambos obtidos da Sigma Aldrich com pureza superior a $99.8 \%$ ) no injetor do cromatógrafo a gás. A pressão no injetor foi mantida constante a $123,6 \mathrm{kPa}$ em todo o período de análise, utilizando o modo splitless por 4 minutos e em seguida uma razão de split igual a 1:10, a temperatura no injetor e detector foram mantidas a $250^{\circ} \mathrm{C}$. A temperatura inicial da coluna foi $50{ }^{\circ} \mathrm{C}$, mantida por 5 minutos, elevando-se a $150^{\circ} \mathrm{C}$ a uma taxa de aquecimento de $10^{\circ} \mathrm{C} \cdot \mathrm{min}^{-1}$, mantendo-se nesta temperatura por mais 1 minuto. 
A determinação do coeficiente de partição fibra-gás $\left(\mathrm{K}_{\mathrm{fg}}\right)$ se deu através da injeção de $1 \mu \mathrm{L}$ do analito em frascos âmbar. A temperatura do sistema foi controlada por um banho termostático (LAUDA, modelo RM 6B). O tempo de extração da fibra no headspace foi determinado, onde a massa extraída não mais variasse com o tempo de exposição na menor temperatura. Sendo de 15 minutos para o etanol e 25 minutos para o 1-butanol. A extração foi realizada para as 4 temperaturas estudadas nas mesmas condições cromatográficas da curva de calibração.

A extração dos analitos através da SPME ocorreu em frascos âmbar, onde $3 \mathrm{~mL}$ de LI foi adicionado juntamente com $1 \mu \mathrm{L}$ de soluto. A mistura permaneceu sob agitação magnética por 40 minutos e por mais 40 minutos em repouso, ambos os tempos definidos experimentalmente. A extração com a fibra ocorreu no tempo determinado. A temperatura do conjunto foi controlada por um controlador do tipo PID com sensor PT-100 com erro de $\pm 0.1 \mathrm{~K}$. Após a extração, a fibra de PDMS foi exposta no injetor do cromatógrafo a gás para a quantificação de material extraído, sendo mantidas as mesmas condições da construção da curva de calibração.

\section{RESULTADOS E DISCUSSÕES}

\subsection{Curva de calibração}

Para os sistemas estudados, as curvas de calibração apresentaram boas correlações. A regressão linear para o etanol apresentou um coeficiente angular de 11042, coeficiente de determinação $\left(\mathrm{R}^{2}\right) 0.9999$ e para o 1-butanol, coeficiente angular 17084 e coeficiente de determinação $\left(\mathrm{R}^{2}\right) 0.9999$.

\subsection{Coeficiente de partição fibra-gás}

Os coeficiente de partição fibra-gás $\left(\mathrm{K}_{\mathrm{fg}}\right)$ foram determinados para os solutos estudados através da Equação 1, onde $\mathrm{V}_{\mathrm{f}}$ é o volume da fibra, $\mathrm{V}_{\mathrm{g}} \mathrm{o}$ volume do headspace do frasco, $\mathrm{n}_{1}{ }^{\mathrm{f}}$ número de mols do soluto absorvido pela fibra e $\mathrm{n}_{1}{ }^{\mathrm{g}}$ número de mols do soluto na fase gasosa do sistema.

$$
K_{f g}=\frac{n_{1}^{f}}{V_{f}} \frac{V_{g}}{n_{1}^{g}}
$$

Para verificar a consistência dos dados experimentais, foi realizada uma regressão linear dos dados, verificando-se a confiabilidade dos dados, como pode ser observado na Figura 1. 


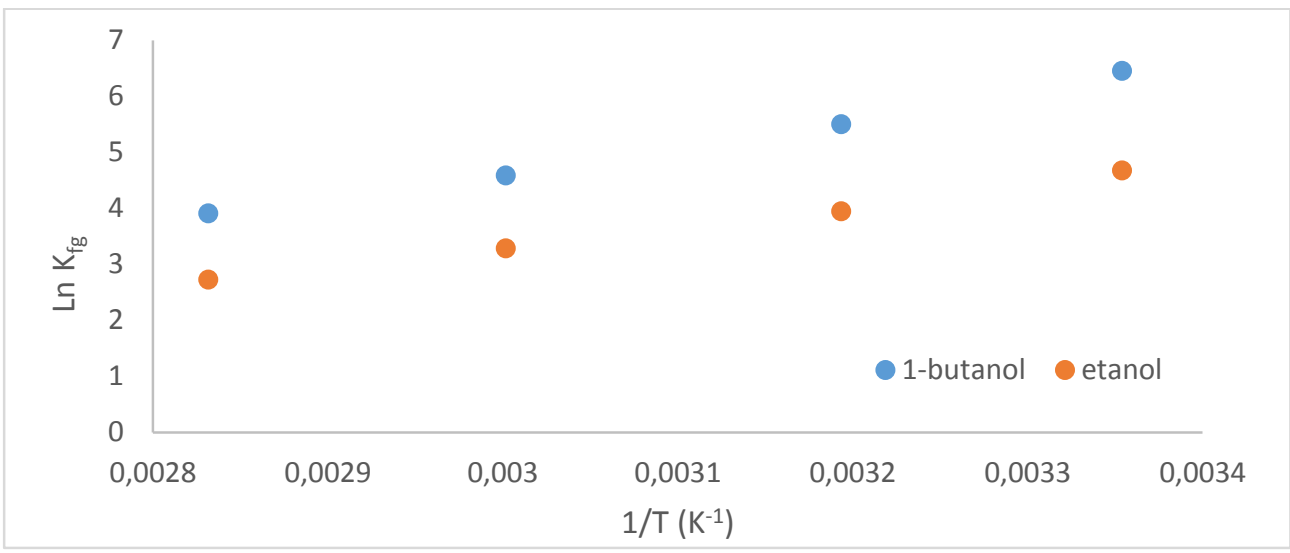

Figura 1 - Linearização do coeficiente de partição fibra-gás $\left(\mathrm{K}_{\mathrm{fg}}\right)$ para verificação da consistência dos dados experimentais.

\subsection{Coeficiente de partição líquido-gás na diluição infinita}

A partir dos dados de coeficiente de partição fibra-gás, pode-se obter o coeficiente de partição líquido-gás $\left(\mathrm{K}_{\mathrm{Lg}}\right)$ para cada soluto estudado através da Equação 2, onde $\mathrm{n}_{\mathrm{o}}{ }^{\mathrm{L}}$ é o número de mols iniciais do soluto em solução e $V_{L}$ o volume de solução.

$$
K_{L g}=\left[K_{f g} V_{f}\left(\frac{n_{o}^{L}}{n_{1}^{f}}-1\right)-V_{g}\right] \frac{1}{V_{L}}
$$

Os valores calculados foram comparados com os valores disponíveis em Dobryakov et al.(2008) e apresentaram boa concordância, com variações menores que $6 \%$ na maioria dos valores encontrados. Os coeficientes de partição liquido-gás teve uma maior variação na temperatura de 333.15 e $353.15 \mathrm{~K}$ para o 1-butanol (15 e $23 \%$, respectivamente). Para garantir a confiabilidade dos dados, foi realizada uma nova linearização, onde foi constatado que os dados são coerentes para as condições estudadas, onde os coeficientes de determinação $\left(\mathrm{R}^{2}\right)$ ficaram em todos os casos maiores que 0,9993 . Os parâmetros determinados podem ser observados na Tabela 1 .

Tabela 1 - Coeficiente de Partição líquido gás na diluição infinita $\left(\mathrm{K}_{\mathrm{i}}{ }^{\infty}\right)$ dos solutos estudados em temperaturas que variaram de 298.15 à $353.15 \mathrm{~K}$.

\begin{tabular}{|c|c|c|c|c|}
\hline \multirow{2}{*}{ Soluto i } & \multicolumn{4}{|c|}{$\mathrm{K}_{\mathrm{i}}^{\infty}$} \\
\hline & $\mathrm{T}(\mathrm{K})$ & $\mathrm{T}(\mathrm{K})$ & $\mathrm{T}(\mathrm{K})$ & $\mathrm{T}(\mathrm{K})$ \\
\hline & 298.15 & 313.15 & 333.15 & 353.15 \\
\hline Etanol & $2214 \pm 27$ & $1088 \pm 37$ & $461 \pm 18$ & $228 \pm 1$ \\
\hline 1-butanol & $7661 \pm 157$ & $3476 \pm 88$ & $1424 \pm 49$ & $690 \pm 33$ \\
\hline
\end{tabular}




\subsection{Coeficiente de atividade na diluição infinita}

Algumas variáveis são necessárias para o correto cálculo do coeficiente de atividade na diluição infinita $\left(\mathrm{\gamma}^{\infty}\right)$ como a densidade do solvente $\left(\rho_{2}{ }^{\mathrm{L}}\right)$ e a pressão de saturação do soluto $\left(\mathrm{P}_{1}{ }^{\text {sat }}\right)$. A densidade do solvente foi calculada de acordo com a correlação proposta por Dobryakov et al. (2008). A pressão de vapor do soluto em cada temperatura foi calculada a partir da equação de Wagner, com os parâmetros encontrados na literatura (Reid et al., 1987).

O segundo coeficiente de virial $\left(\mathrm{B}_{11}\right)$ foi determinado pelo método de Tsonopoulos e o volume molar do soluto $\left(\mathrm{v}_{1}{ }^{0}\right)$ foi determinado pela equação empírica de Rackett. As equações e os parâmetro estão disponíveis na literatura (Reid et al., 1987).

Através da Equação 3 e dos parâmetros determinados, pode-se determinar o coeficiente de atividade na diluição infinita dos compostos, onde $\mathrm{T}$ é a temperatura, $\mathrm{M}_{2}$ massa molar do solvente e $\mathrm{R}$ a constante dos gases. Os valores encontrados estão demonstrados na Tabela 2.

$$
\ln \gamma_{1}^{\infty}=\ln \left(\frac{\rho_{2}^{L} R T}{K_{L g} P_{1}^{s a t} M_{2}}\right)-\frac{P_{1}^{s a t}\left(B_{11}-v_{1}^{o}\right)}{R T}
$$

Tabela 2 - Coeficiente de atividade na diluição infinita $\left(\mathrm{y}_{\mathrm{i}}^{\infty}\right)$ dos solutos estudados em temperaturas que variaram de 298.15 à $353.15 \mathrm{~K}$.

\begin{tabular}{|c|c|c|c|c|}
\hline Soluto i & \multicolumn{4}{|c|}{$\mathrm{\gamma i}^{\infty}$} \\
\hline & $\mathrm{T}(\mathrm{K})$ & $\mathrm{T}(\mathrm{K})$ & $\mathrm{T}(\mathrm{K})$ & $\mathrm{T}(\mathrm{K})$ \\
\hline & 298.15 & 313.15 & 333.15 & 353.15 \\
\hline Etanol & $0.69 \pm 0.01$ & $0.65 \pm 0.02$ & $0.63 \pm 0.02$ & $0.56 \pm 0.01$ \\
\hline 1-butanol & $1.75 \pm 0.04$ & $1.46 \pm 0.04$ & $1.16 \pm 0.04$ & $0.94 \pm 0.05$ \\
\hline
\end{tabular}

Os dados encontrados foram comparados com a literatura. Para o etanol, os valores apresentaram desvios menores que $8 \%$. Para o 1-butanol, nas temperaturas de 298.15 e $313.15 \mathrm{~K}$, o desvio ficou menor que $4 \%$. Nas temperaturas de 333.15 e $353.15 \mathrm{~K}$ os desvios foram de 15 e $23 \%$ respectivamente. $\mathrm{O}$ valor se deve ao fato da diferença nos coeficiente de partição líquido-gás encontrados com o valores da literatura. Por meio de linearização, comparou-se os dois resultados, que podem ser observados na Figura 2 e na Figura 3. 


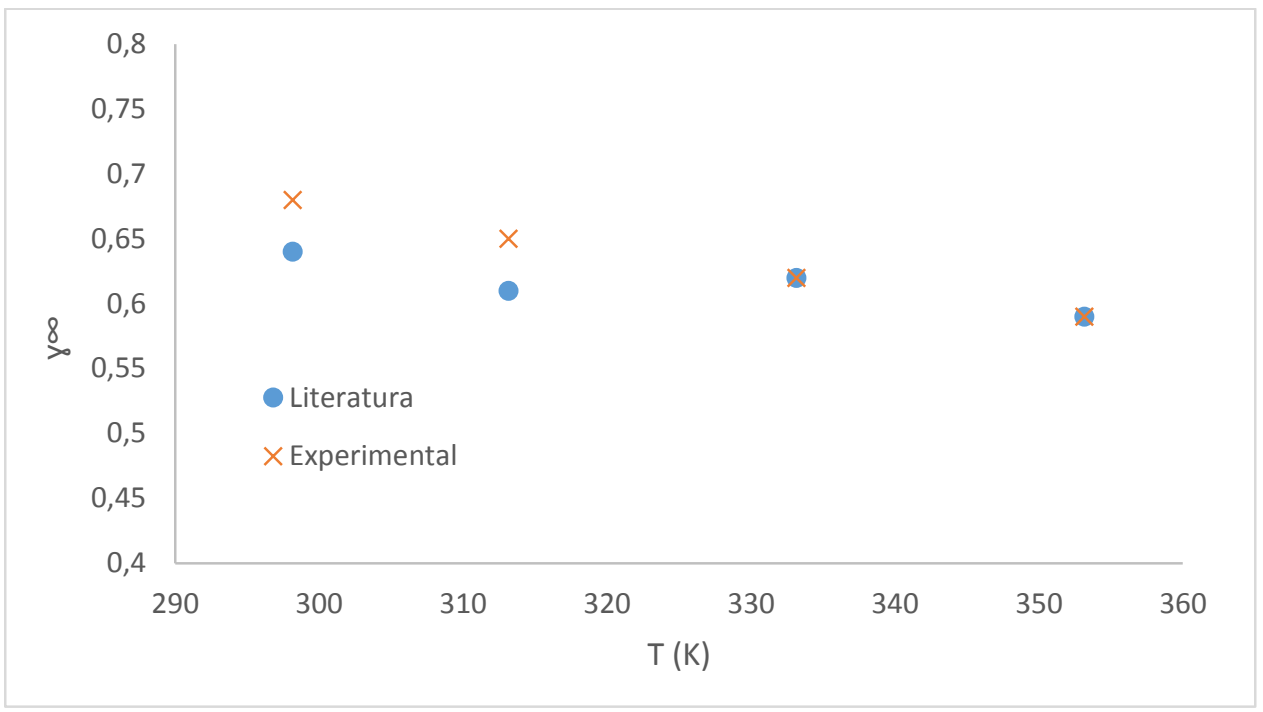

Figura 2 - Linearização do coeficiente de atividade na diluição infinita $\left(\mathrm{\gamma}_{1}^{\infty}\right)$ do etanol em comparação aos valores da literatura (Dobryakov et al.,2008), nas temperaturas estudadas.

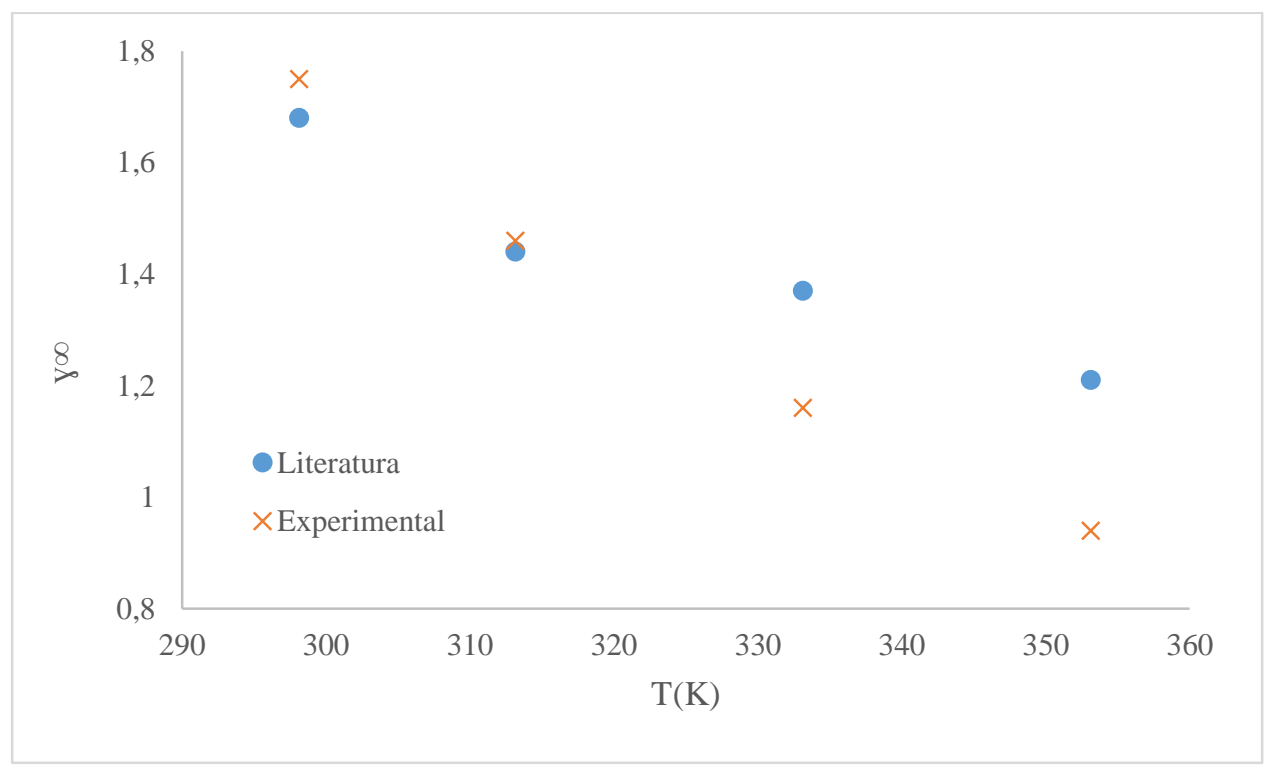

Figura 3 - Linearização do coeficiente de atividade na diluição infinita $\left(\mathrm{y}^{\infty}{ }^{\infty}\right)$ do 1-butanol em comparação aos valores da literatura (Dobryakov et al.,2008), nas temperaturas estudadas.

Para os sistemas estudados, o coeficiente de atividade na diluição infinita reduziu o valor com a elevação da temperatura. Este comportamento indica que as forças de repulsão solvente-soluto diminuíram. Tal comportamento é mais comum em alcanos e ciclocanos do que em álcoois. Uma explicação seria a presença de elétrons deslocalizados que podem interagir com o cátion e/ou ânion do liquido iônico (Dobryakov et al.,2008). 


\section{9 a 22 de outubro de 2014 \\ Florianópolis/SC}

Através da Figura 2 e da Figura 3, nota-se que o coeficiente de atividade aumenta com o aumento da cadeia de carbonos. Devido a inexistência de mais dados na literatura, a comparação se dá somente através do trabalho realizado por Dobryakov et al. (2008). Mais estudos envolvendo líquidos iônicos necessitam ser realizados devido a pequena biblioteca de dados disponível atualmente.

\section{CONCLUSÕES}

Os coeficientes de atividades na diluição infinita determinados para os sistemas estudados apresentaram duas características interessantes: o aumento da temperatura diminui o coeficiente de atividade, pois aumenta a atração entre soluto-solvente; a redução da cadeia de carbonos nos álcoois estudados em metilsulfato de 1-butil-3-metilimidazolíneo reduz o coeficiente de atividade, entre outros fatores, pelas interações de elétrons deslocalizados com o cátion e/ou ânions do líquido iônico.

A SPME apresentou bons resultados quando comparada com a literatura, apresentando valores que variaram de 0.8 a 23\%. A grande variação se deu nas diferenças entre os coeficientes de partição na diluição infinita. Para garantir a credibilidade dos dados foi realizada uma linearização, que permitiu constatar a coerência entre todos os dados experimentais deste trabalho.

A microextração em fase sólida mostrou-se como uma técnica eficiente, rápida e de baixo custo, garantindo valores satisfatórios para a determinação de coeficiente de atividade na diluição infinita.

\section{REFERÊNCIAS}

DOBRYAKOV, Y.G.; TUMA, D.; MAURER, G.; Activity Coefficients at Infinite Dilution of Alkanols in the Ionic Liquids 1-Butyl-3-methylimidazolium Hexafluorophosphate, 1-Butyl-3methylimidazolium Methyl Sulfate and 1-Hexyl-3-methylimidazolium Bis(trifluoromethylsulfonyl) Amide Using the Dilutor Technique, J. Chem. Eng. Data, v. 53, p. 2154-2162, 2008.

FONSECA, D. B.; COELHO, G. L. V.; Determinação do coeficiente de atividade na diluição infinita $(\gamma \infty)$ através da microextração em fase sólida (SPME), Quim. Nova, v. 30, p. 1606-1608, 2007

FURTADO, F.A.; COELHO, G.L.V.; Determinação do coeficiente de atividade em diluição infinita de hidrocarbonetos em furfural a 298,15 k por SPME-GC/FID, Quim. Nova, v. 33, p. 19051909, 2010.

FURTADO, F.A.; COELHO, G.L.V.; Determination of infinite dilution activity coefficients using HS-SPME/GC/FID for hydrocarbons in furfural at temperatures of $(298.15,308.15$, and 318.15) K, J. Chem. Thermo., v. 49, p. 119-127, 2012

GREAVES, T.L.; DRUMMOND, C.J. Protic ionic liquids: Properties and Aplications, Chem. Rev., v. 108, p. 206-237, 2008.

HUANG, H.J.; RAMASWAMY, S.; TSCHIRNER, U.W.; RAMARAO, B.V.; A review of separation technologies in current and future biorefineries, Sep. and Pur. Tech., v. 62, p. 1-21 , 2008. 
KRUMMEN, M.; WASSERSCHEID, P.; GMEHLING, J.; Measurement of Activity Coefficients at Infinite Dilution in Ionic, J. Chem. Eng. Data, v. 47, p. 1411-1417, 2002

REID, R.C.; PRAUSNITZ, J.M.; POLING, B.E.; The Properties of Gases and Liquids, 4 ${ }^{\mathrm{a}}$ Edição, New York, Mc Graw Hill, 1987.

ZHANG, Z.; PAWLISZYN, J.; Studying Activity Coefficients of Probe Solutes in Selected Liquid Polymer Coatings Using Solid Phase Microextraction, J. Phys. Chem., v. 100, p. 17648 -17645, 1996. 